\title{
Cubic-Quartic Optical Solitons in Bragg Gratings Fibers for Perturbed NLSE Having Parabolic- Nonlocal Law of Refractive Index Using Two Integration Schemes
}

\section{Elsayed M. E. Zayed}

Zagazig University Faculty of Science

Mohamed E. M. Alngar ( $\sim$ mohamed.alngar2010@yahoo.com )

Zagazig University Faculty of Science https://orcid.org/0000-0002-5436-7268

\section{Research Article}

Keywords: Jacobi elliptic function solutions, The extended auxiliary equation method, The addendum to Kudryashov0s method, Fiber Bragg gratings, Cubic-quartic, Parabolic law, Nonlocal law.

Posted Date: June 22nd, 2021

DOl: https://doi.org/10.21203/rs.3.rs-615796/v1

License: (c) (i) This work is licensed under a Creative Commons Attribution 4.0 International License. Read Full License

Version of Record: A version of this preprint was published at Optical and Quantum Electronics on August 6th, 2021. See the published version at https://doi.org/10.1007/s11082-021-03145-x. 


\title{
Cubic-quartic optical solitons in Bragg gratings fibers for perturbed NLSE having parabolic-nonlocal law of refractive index using two integration schemes
}

\author{
Elsayed M. E. Zayed, Reham.M.A.Shohib \& Mohamed E. M. Alngar \\ Mathematics Department, Faculty of Science, Zagazig University, \\ Zagazig-44519, Egypt
}

\begin{abstract}
The optical solitons in Bragg gratings fibers for perturbed NLSE having cubic-quartic dispersive reflectivity with parabolic-nonlocal combo law of refractive index are studied. The extended auxiliary equation method and the addendum to Kudryashov's method are introduced. The existence criteria for such solitons are indicated.
\end{abstract}

Keywords: Jacobi elliptic function solutions; The extended auxiliary equation method; The addendum to Kudryashov's method ; Fiber Bragg gratings; Cubic-quartic; Parabolic law; Nonlocal law.

PACS Numbers: 02.30.Jr; 02.30.Ik; 05.45.Yv

OCIS Codes: 060.2310; 060.4510; 060.5530; 190.3270; 190.4370

\section{Introduction}

In the past few decades [1-22], the study of optical solitons in optical fibers, PCF, metamaterials, optical couplers, fibers with Bragg gratings is going on strong and steady. Lately, when the chromatic dispersion (CD) gets low, the definition of cubic-quartic (CQ) solitons have emerged when it was realized that the delicate balance between CD and self-phase modulation (SPM) grows feeble. In that case, to boost up the much-needed balance, it is necessary to compensate for the low count of CD by introducing third-order dispersion (3OD) and fourth-order dispersion (4OD) terms. Together, these constitute CQ dispersive effects. For Bragg gratings fibers, it is the dispersive reflectivity, which originally stems from $\mathrm{CD}$, now comes from $\mathrm{CQ}$ dispersion. In this paper, the coupled system of cubic-quartic nonlinear Schrödinger equation (CQ-NLSE) in Bragg gratings fibers with parabolic-nonlocal combo law of refractive index will be discussed for the first time by using two different methods mentioned in the abstract. Today's work will address the same problem with two fresh approaches. These are the extended auxiliary equation method and the 
addendum to Kudryashov's method. These methods will lead to the emergence of Jacobi elliptic function solutions, bright, dark and singular solitons from the governing model. The essential details are jotted with the existence criteria for such solitons, after a quick into to the model.

\subsection{Governing model}

The coupled cubic-quartic perturbed NLSE in Bragg gratings fibers with parabolic-nonlocal law nonlinearity is written, for the first time, as

$$
\begin{gathered}
i u_{t}+i a_{1} v_{x x x}+b_{1} v_{x x x x}+\left(c_{1}|u|^{2}+d_{1}|v|^{2}\right) u+\left(\xi_{1}|u|^{4}+\eta_{1}|u|^{2}|v|^{2}+\zeta_{1}|v|^{4}\right) u \\
+\left[f_{1}\left(|u|^{2}\right)_{x x}+g_{1}\left(|v|^{2}\right)_{x x}\right] u+i \alpha_{1} u_{x}+\beta_{1} v+\sigma_{1} u^{*} v^{2}=i\left[\gamma_{1}\left(|u|^{2} u\right)_{x}+\theta_{1}\left(|u|^{2}\right)_{x} u+\rho_{1}|u|^{2} u_{x}\right],
\end{gathered}
$$

and

$$
\begin{gathered}
i v_{t}+i a_{2} u_{x x x}+b_{2} u_{x x x x}+\left(c_{2}|v|^{2}+d_{2}|u|^{2}\right) v+\left(\xi_{2}|v|^{4}+\eta_{2}|v|^{2}|u|^{2}+\zeta_{2}|u|^{4}\right) v \\
+\left[f_{2}\left(|v|^{2}\right)_{x x}+g_{2}\left(|u|^{2}\right)_{x x}\right] v+i \alpha_{2} v_{x}+\beta_{2} u+\sigma_{2} v^{*} u^{2}=i\left[\gamma_{2}\left(|v|^{2} v\right)_{x}+\theta_{2}\left(|v|^{2}\right)_{x} v+\rho_{2}|v|^{2} v_{x}\right]
\end{gathered}
$$

where $a_{l}, b_{l}, c_{l}, d_{l}, \xi_{l}, \eta_{l}, \zeta_{l}, f_{l}, g_{l}, \alpha_{l}, \beta_{l}, \sigma_{l}, \gamma_{l}, \theta_{l}$ and $\rho_{l},(l=1,2)$ are real parameters such that $i=$ $\sqrt{-1}$. Here, $u(x, t), v(x, t)$ are the complex wave profiles. The coefficients of $a_{l}, b_{l}$ are third order dispersion(3OD) and fourth order dispersion (4OD), respectively. The parameters $c_{l}, \xi_{l}, f_{l}$ represent the self-phase modulation (SPM) coefficients, while the cross-phase modulation (XPM) effect comes from the coefficients $d_{l}, \eta_{l}, \zeta_{l}$ and $g_{l}$. The parameters $\alpha_{l}, \beta_{l}$ and $\sigma_{l}$ are the coefficients of inter-modal dispersion (IMD), detuning parameter and four-wave mixing effect (4WM) for Kerr part of the nonlinearity, respectively, while, $\gamma_{l}$ is the coefficient of self-steepening (SS) term. Lastly, the parameters $\theta_{l}, \rho_{l}$ are the coefficients of nonlinear dispersion terms. The system (1) and (2) is a manifested version of the standard model. That is the well-known NLSE model in fiber Bragg gratings with chromatic dispersion (CD), that is structured as [22]:

$$
\begin{aligned}
i u_{t}+E_{1} v_{x x}+\left(c_{1}|u|^{2}+d_{1}|v|^{2}\right) u+ & \left(\xi_{1}|u|^{4}+\eta_{1}|u|^{2}|v|^{2}+\zeta_{1}|v|^{4}\right) u+\left[f_{1}\left(|u|^{2}\right)_{x x}+g_{1}\left(|v|^{2}\right)_{x x}\right] u \\
& +i \alpha_{1} u_{x}+\beta_{1} v+\sigma_{1} u^{*} v^{2}=0
\end{aligned}
$$

and

$$
\begin{aligned}
i v_{t}+E_{2} u_{x x}+\left(c_{2}|v|^{2}+d_{2}|u|^{2}\right) v+ & \left(\xi_{2}|v|^{4}+\eta_{2}|u|^{2}|v|^{2}+\zeta_{2}|u|^{4}\right) v+\left[f_{2}\left(|v|^{2}\right)_{x x}+g_{2}\left(|u|^{2}\right)_{x x}\right] v \\
& +i \alpha_{2} v_{x}+\beta_{2} u+\sigma_{2} v^{*} u^{2}=0
\end{aligned}
$$

where $E_{1}$ and $E_{2}$ are the coefficients of chromatic dispersion (CD). In the system (1) and (2), it is this CD that is replaced by $3 \mathrm{OD}$ and 4OD, which formulate the dispersion effects.

The objective of this paper is to apply the extended auxiliary equation method and the addendum to Kudryashov's method to find the bright, dark and singular solitons solutions as well as the 
Jacobi elliptic function solutions of the coupled system (1) and (2).

The organization of this article can be written as: the mathematical preliminaries are discussed in Section 2. The extended auxiliary equation method is applied to the coupled system (1) and (2) in Section 3. The addendum to Kudryashov's method is applied to the same coupled system in Section 4. Lastly, conclusions are given in Section 5.

\section{Mathematical preliminaries}

In order to recover solitons of the CQ-NLSE in fiber Bragg gratings with parabolic non-local law nonlinearity, we set

$$
\begin{gathered}
u(x, t)=P_{1}(\xi) \exp [i \phi(x, t)], \\
v(x, t)=P_{2}(\xi) \exp [i \phi(x, t)],
\end{gathered}
$$

and

$$
\xi=x-C t, \phi(x, t)=-\kappa x+\omega t+\theta_{0},
$$

where $C, \kappa, \omega$ and $\theta_{0}$ are all non zero parameters. Here, $C$ is the velocity of soliton, $\kappa$ is the frequency of soliton, $\omega$ is the wave number of the soliton and finally, $\theta_{0}$ is the phase parameter, while $P_{1}(\xi), P_{2}(\xi)$ and $\phi(x, t)$ are real functions representing the amplitude portion of the soliton and the phase component of the soliton, respectively. If we substitute (5) and (6) into Eqs. (1) and (2) and separate the real and imaginary parts, we deduce that the real parts are

$$
\begin{gathered}
b_{1} P_{2}^{(4)}+3 \kappa\left(a_{1}-2 b_{1} \kappa\right) P_{2}^{\prime \prime}+\left(\alpha_{1} \kappa-\omega\right) P_{1}+\left[\kappa^{3}\left(b_{1} \kappa-a_{1}\right)+\beta_{1}\right] P_{2}+\left(c_{1}-\gamma_{1} \kappa-\rho_{1} \kappa\right) P_{1}^{3} \\
+2\left[f_{1}\left(P_{1} P_{1}^{\prime 2}+P_{1}^{2} P_{1}^{\prime \prime}\right)+g_{1}\left(P_{1} P_{2}^{\prime 2}+P_{1} P_{2} P_{2}^{\prime \prime}\right)\right]+\left(d_{1}+\sigma_{1}\right) P_{1} P_{2}^{2}+\xi_{1} P_{1}^{5}+\eta_{1} P_{1}^{3} P_{2}^{2}+\zeta_{1} P_{1} P_{2}^{4}=0 \\
b_{2} P_{1}^{(4)}+3 \kappa\left(a_{2}-2 b_{2} \kappa\right) P_{1}^{\prime \prime}+\left(\alpha_{2} \kappa-\omega\right) P_{2}+\left[\kappa^{3}\left(b_{2} \kappa-a_{2}\right)+\beta_{2}\right] P_{1}+\left(c_{2}-\gamma_{2} \kappa-\rho_{2} \kappa\right) P_{2}^{3} \\
+2\left[f_{2}\left(P_{2} P_{2}^{\prime 2}+P_{2}^{2} P_{2}^{\prime \prime}\right)+g_{2}\left(P_{2} P_{1}^{\prime 2}+P_{2} P_{1} P_{1}^{\prime \prime}\right)\right]+\left(d_{2}+\sigma_{2}\right) P_{2} P_{1}^{2}+\xi_{2} P_{2}^{5}+\eta_{2} P_{2}^{3} P_{1}^{2}+\zeta_{2} P_{2} P_{1}^{4}=0,
\end{gathered}
$$

and the imaginary parts are

$$
\begin{aligned}
& \left(a_{1}-4 b_{1} \kappa\right) P_{2}^{\prime \prime \prime}-\left(3 a_{1}-4 b_{1} \kappa\right) \kappa^{2} P_{2}^{\prime}+\left(\alpha_{1}-C\right) P_{1}^{\prime}-\left(3 \gamma_{1}+2 \theta_{1}+\rho_{1}\right) P_{1}^{2} P_{1}^{\prime}=0 \\
& \left(a_{2}-4 b_{2} \kappa\right) P_{1}^{\prime \prime \prime}-\left(3 a_{2}-4 b_{2} \kappa\right) \kappa^{2} P_{1}^{\prime}+\left(\alpha_{2}-C\right) P_{2}^{\prime}-\left(3 \gamma_{2}+2 \theta_{2}+\rho_{2}\right) P_{2}^{2} P_{2}^{\prime}=0 .
\end{aligned}
$$

Setting

$$
P_{2}(\xi)=\chi P_{1}(\xi)
$$

where $\chi$ is a constant such that $\chi \neq 0,1$. Now, Eqs.(7) - (10) become

$$
\begin{gathered}
b_{1} \chi P_{1}^{(4)}+3 \kappa \chi\left(a_{1}-2 b_{1} \kappa\right) P_{1}^{\prime \prime}+2\left(f_{1}+\chi^{2} g_{1}\right)\left(P_{1} P_{1}^{\prime 2}+P_{1}^{2} P_{1}^{\prime \prime}\right) \\
+\left[\alpha_{1} \kappa-\omega+\chi \kappa^{3}\left(b_{1} \kappa-a_{1}\right)+\chi \beta_{1}\right] P_{1}+\left[c_{1}-\gamma_{1} \kappa-\rho_{1} \kappa+\chi^{2}\left(d_{1}+\sigma_{1}\right)\right] P_{1}^{3} \\
+\left(\xi_{1}+\eta_{1} \chi^{2}+\chi^{4} \zeta_{1}\right) P_{1}^{5}=0
\end{gathered}
$$




$$
\begin{gathered}
b_{2} P_{1}^{(4)}+3 \kappa\left(a_{2}-2 b_{2} \kappa\right) P_{1}^{\prime \prime}+2 \chi\left(\chi^{2} f_{2}+g_{2}\right)\left(P_{1} P_{1}^{\prime 2}+P_{1}^{2} P_{1}^{\prime \prime}\right) \\
+\left[\chi\left(\alpha_{2} \kappa-\omega\right)+\kappa^{3}\left(b_{2} \kappa-a_{2}\right)+\beta_{2}\right] P_{1}+\chi\left[\chi^{2}\left(c_{2}-\gamma_{2} \kappa-\rho_{2} \kappa\right)+d_{2}+\sigma_{2}\right] P_{1}^{3} \\
+\chi\left(\chi^{4} \xi_{2}+\chi^{2} \eta_{2}+\zeta_{2}\right) P_{1}^{5}=0
\end{gathered}
$$

and

$$
\begin{gathered}
\chi\left(a_{1}-4 b_{1} \kappa\right) P_{1}^{\prime \prime \prime}+\left[\alpha_{1}-C-\chi\left(3 a_{1}-4 b_{1} \kappa\right) \kappa^{2}\right] P_{1}^{\prime}-\left(3 \gamma_{1}+2 \theta_{1}+\rho_{1}\right) P_{1}^{2} P_{1}^{\prime}=0 \\
\left(a_{2}-4 b_{2} \kappa\right) P_{1}^{\prime \prime \prime}+\left[\chi\left(\alpha_{2}-C\right)-\left(3 a_{2}-4 b_{2} \kappa\right) \kappa^{2}\right] P_{1}^{\prime}-\chi^{3}\left(3 \gamma_{2}+2 \theta_{2}+\rho_{2}\right) P_{1}^{2} P_{1}^{\prime}=0 .
\end{gathered}
$$

Integrating Eqs.(14) and (15) with zero-integration constants, we have

$$
\begin{gathered}
\chi\left(a_{1}-4 b_{1} \kappa\right) P_{1}^{\prime \prime}+\left[\alpha_{1}-C-\chi\left(3 a_{1}-4 b_{1} \kappa\right) \kappa^{2}\right] P_{1}-\frac{1}{3}\left(3 \gamma_{1}+2 \theta_{1}+\rho_{1}\right) P_{1}^{3}=0, \\
\left(a_{2}-4 b_{2} \kappa\right) P_{1}^{\prime \prime}+\left[\chi\left(\alpha_{2}-C\right)-\left(3 a_{2}-4 b_{2} \kappa\right) \kappa^{2}\right] P_{1}-\frac{1}{3} \chi^{3}\left(3 \gamma_{2}+2 \theta_{2}+\rho_{2}\right) P_{1}^{3}=0 .
\end{gathered}
$$

Setting the coefficients of the linearly independent functions of Eqs. (16) and (17) to zero, yields

$$
\begin{gathered}
\kappa=\frac{a_{l}}{4 b_{l}}, \quad l=1,2, \quad a_{1} b_{2}=a_{2} b_{1}, \\
C=\alpha_{1}-\chi\left(3 a_{1}-4 b_{1} \kappa\right) \kappa^{2}, \\
C=\frac{\chi \alpha_{2}-\left(3 a_{2}-4 b_{2} \kappa\right) \kappa^{2}}{\chi},
\end{gathered}
$$

and

$$
\begin{aligned}
& 3 \gamma_{1}+2 \theta_{1}+\rho_{1}=0, \\
& 3 \gamma_{2}+2 \theta_{2}+\rho_{2}=0 .
\end{aligned}
$$

From (19) and (20), one gets the following constraint condition:

$$
\chi\left(\alpha_{1}-\alpha_{2}\right)-3 \kappa^{2}\left(a_{1} \chi^{2}-a_{2}\right)+4 \kappa^{3}\left(b_{1} \chi^{2}-b_{2}\right)=0 .
$$

Eqs. (12) and (13) have the same form under the constraint conditions:

$$
\begin{aligned}
b_{1} \chi & =b_{2}, \\
\chi\left(a_{1}-2 b_{1} \kappa\right) & =a_{2}-2 b_{2} \kappa, \\
f_{1}+\chi^{2} g_{1} & =\chi\left(\chi^{2} f_{2}+g_{2}\right), \\
\alpha_{1} \kappa-\omega+\chi \kappa^{3}\left(b_{1} \kappa-a_{1}\right)+\chi \beta_{1} & =\chi\left(\alpha_{2} \kappa-\omega\right)+\kappa^{3}\left(b_{2} \kappa-a_{2}\right)+\beta_{2}, \\
c_{1}-\gamma_{1} \kappa-\rho_{1} \kappa+\chi^{2}\left(d_{1}+\sigma_{1}\right) & =\chi\left[\chi^{2}\left(c_{2}-\gamma_{2} \kappa-\rho_{2} \kappa\right)+d_{2}+\sigma_{2}\right], \\
\xi_{1}+\eta_{1} \chi^{2}+\chi^{4} \zeta_{1} & =\chi\left(\chi^{4} \xi_{2}+\chi^{2} \eta_{2}+\zeta_{2}\right) .
\end{aligned}
$$


Consequently, we deduce that

$$
\begin{aligned}
& \omega=\frac{\left(\alpha_{1}-\chi \alpha_{2}\right) \kappa+\chi \beta_{1}-\beta_{2}}{1-\chi}, \\
& \chi=\frac{b_{2}}{b_{1}}=\frac{a_{2}-2 b_{2} \kappa}{a_{1}-2 b_{1} \kappa},
\end{aligned}
$$

provided $a_{1} \neq a_{2}$ and $b_{1} \neq b_{2}$. Eq. (12) can be rewritten in the form:

$$
P_{1}^{(4)}+L_{1} P_{1}^{\prime \prime}+L_{2}\left(P_{1} P_{1}^{\prime 2}+P_{1}^{2} P_{1}^{\prime \prime}\right)+L_{3} P_{1}+L_{4} P_{1}^{3}+L_{5} P_{1}^{5}=0,
$$

where

$$
\begin{gathered}
L_{1}=6 \kappa^{2}, L_{2}=\frac{2\left(f_{1}+\chi^{2} g_{1}\right)}{b_{1} \chi}, L_{3}=\frac{\alpha_{1} \kappa-\omega+\chi \kappa^{3}\left(b_{1} \kappa-a_{1}\right)+\chi \beta_{1}}{b_{1} \chi}, \\
L_{4}=\frac{c_{1}-\gamma_{1} \kappa-\rho_{1} \kappa+\chi^{2}\left(d_{1}+\sigma_{1}\right)}{b_{1} \chi}, L_{5}=\frac{\xi_{1}+\eta_{1} \chi^{2}+\chi^{4} \zeta_{1}}{b_{1} \chi}
\end{gathered}
$$

provided $b_{1} \chi \neq 0$ and $L_{1}>0$. Balancing $P_{1}^{(4)}$ with $P_{1}^{5}$ in (31), yields the balance number $N=1$. The problem now is to solve Eq. (31) using the following two schemes.

\section{The extended auxiliary equation method}

According to this method [15-18], we assume that Eq.(31) has the formal solution

$$
P_{1}(\xi)=A_{0}+A_{1} F(\xi)+A_{2} F^{2}(\xi),
$$

where $A_{0}, A_{1}$ and $A_{2}$ are constants to be determined, such that $A_{2} \neq 0$, while the function $F(\xi)$ satisfies the following first order equation:

$$
F^{2}(\xi)=C_{0}+C_{2} F^{2}(\xi)+C_{4} F^{4}(\xi)+C_{6} F^{6}(\xi),
$$

where $C_{j}(j=0,2,4,6)$ are constants to be determined. It is well known that Eq.(34) has the following solution:

$$
F(\xi)=\frac{1}{2}\left[-\frac{C_{4}}{C_{6}}(1 \pm f(\xi))\right]^{\frac{1}{2}},
$$

where $f(\xi)$ could be expressed through the Jacobi elliptic functions $\operatorname{sn}(\xi, m), \operatorname{cn}(\xi, m), \operatorname{dn}(\xi, m)$ and so on. Here $0<m<1$ is the modulus of the Jacobi elliptic functions. Substituting (33) along with (34) into Eq.(31), collecting the coefficients of each power $F^{l}(\xi)\left(F^{\prime}(\xi)\right)^{j},(l=0,1,2, . ., 10$, $j=0,1)$ and setting these coefficients to zero, we have a set of algebraic equations which can be solved using the Maple to obtain the following results:

$$
\begin{gathered}
L_{2}=-\frac{5 \sqrt{6 L_{5}}}{3}, L_{3}=-A_{0}^{4} L_{5}+\frac{1}{3} A_{0}\left(L_{1} A_{0}+4 A_{2} C_{0}\right) \sqrt{6 L_{5}}, L_{4}=-\frac{L_{1} \sqrt{6 L_{5}}}{3}, C_{0}=C_{0}, \\
C_{2}=\frac{A_{0}^{2} \sqrt{6 L_{5}}-L_{1}}{4}, C_{4}=\frac{A_{0} A_{2} \sqrt{6 L_{5}}}{6}, C_{6}=\frac{A_{2}^{2} \sqrt{6 L_{5}}}{24}, A_{0}=A_{0}, A_{1}=0, A_{2}=A_{2},
\end{gathered}
$$


provided $L_{5}>0$.From (33), (35) and (36), then we have the solutions:

$$
P_{1}(\xi)=\mp A_{0} f(\xi) .
$$

We have the following families of Jacobi elliptic functions solutions of Eqs.(1) and (2):

Family-1. If $C_{0}=\frac{C_{4}^{3}\left(m^{2}-1\right)}{32 C_{6}^{2} m^{2}}, C_{2}=\frac{C_{4}^{2}\left(5 m^{2}-1\right)}{16 C_{6} m^{2}}, C_{6}>0$, then

$$
u(x, t)=\left(\frac{6 m^{4} L_{1}^{2}}{L_{5}\left(m^{2}+1\right)^{2}}\right)^{\frac{1}{4}} \operatorname{sn}\left(\sqrt{\frac{L_{1}}{m^{2}+1}} \xi, m\right) \mathrm{e}^{i\left(-\kappa x+\omega t+\theta_{0}\right)},
$$

and

$$
v(x, t)=\chi\left(\frac{6 m^{4} L_{1}^{2}}{L_{5}\left(m^{2}+1\right)^{2}}\right)^{\frac{1}{4}} \operatorname{sn}\left(\sqrt{\frac{L_{1}}{m^{2}+1}} \xi, m\right) \mathrm{e}^{i\left(-\kappa x+\omega t+\theta_{0}\right)}
$$

or

$$
u(x, t)=\left(\frac{6 L_{1}^{2}}{L_{5}\left(m^{2}+1\right)^{2}}\right)^{\frac{1}{4}} \mathrm{~ns}\left(\sqrt{\frac{L_{1}}{m^{2}+1}} \xi, m\right) \mathrm{e}^{i\left(-\kappa x+\omega t+\theta_{0}\right)},
$$

and

$$
v(x, t)=\chi\left(\frac{6 L_{1}^{2}}{L_{5}\left(m^{2}+1\right)^{2}}\right)^{\frac{1}{4}} \mathrm{~ns}\left(\sqrt{\frac{L_{1}}{m^{2}+1}} \xi, m\right) \mathrm{e}^{i\left(-\kappa x+\omega t+\theta_{0}\right)} .
$$

In particular, if $m \rightarrow 1^{-}$, then we have the dark soliton solutions:

$$
u(x, t)=\left(\frac{3 L_{1}^{2}}{2 L_{5}}\right)^{\frac{1}{4}} \tanh \left(\sqrt{\frac{L_{1}}{2} \xi}\right) \mathrm{e}^{i\left(-\kappa x+\omega t+\theta_{0}\right)},
$$

and

$$
v(x, t)=\chi\left(\frac{3 L_{1}^{2}}{2 L_{5}}\right)^{\frac{1}{4}} \tanh \left(\sqrt{\frac{L_{1}}{2} \xi}\right) \mathrm{e}^{i\left(-\kappa x+\omega t+\theta_{0}\right)},
$$

as well as the singular soliton solution:

$$
u(x, t)=\left(\frac{3 L_{1}^{2}}{2 L_{5}}\right)^{\frac{1}{4}} \operatorname{coth}\left(\sqrt{\frac{L_{1}}{2}} \xi\right) \mathrm{e}^{i\left(-\kappa x+\omega t+\theta_{0}\right)},
$$

and

$$
v(x, t)=\chi\left(\frac{3 L_{1}^{2}}{2 L_{5}}\right)^{\frac{1}{4}} \operatorname{coth}\left(\sqrt{\frac{L_{1}}{2} \xi}\right) \mathrm{e}^{i\left(-\kappa x+\omega t+\theta_{0}\right)},
$$

while if $m \rightarrow 0^{+}$, then we have the periodic solutions:

$$
u(x, t)=\left(\frac{6 L_{1}^{2}}{L_{5}}\right)^{\frac{1}{4}} \csc \left(\sqrt{L_{1}} \xi\right) \mathrm{e}^{i\left(-\kappa x+\omega t+\theta_{0}\right)},
$$


and

$$
v(x, t)=\chi\left(\frac{6 L_{1}^{2}}{L_{5}}\right)^{\frac{1}{4}} \csc \left(\sqrt{L_{1}} \xi\right) \mathrm{e}^{i\left(-\kappa x+\omega t+\theta_{0}\right)}
$$

provided $L_{5}>0$.

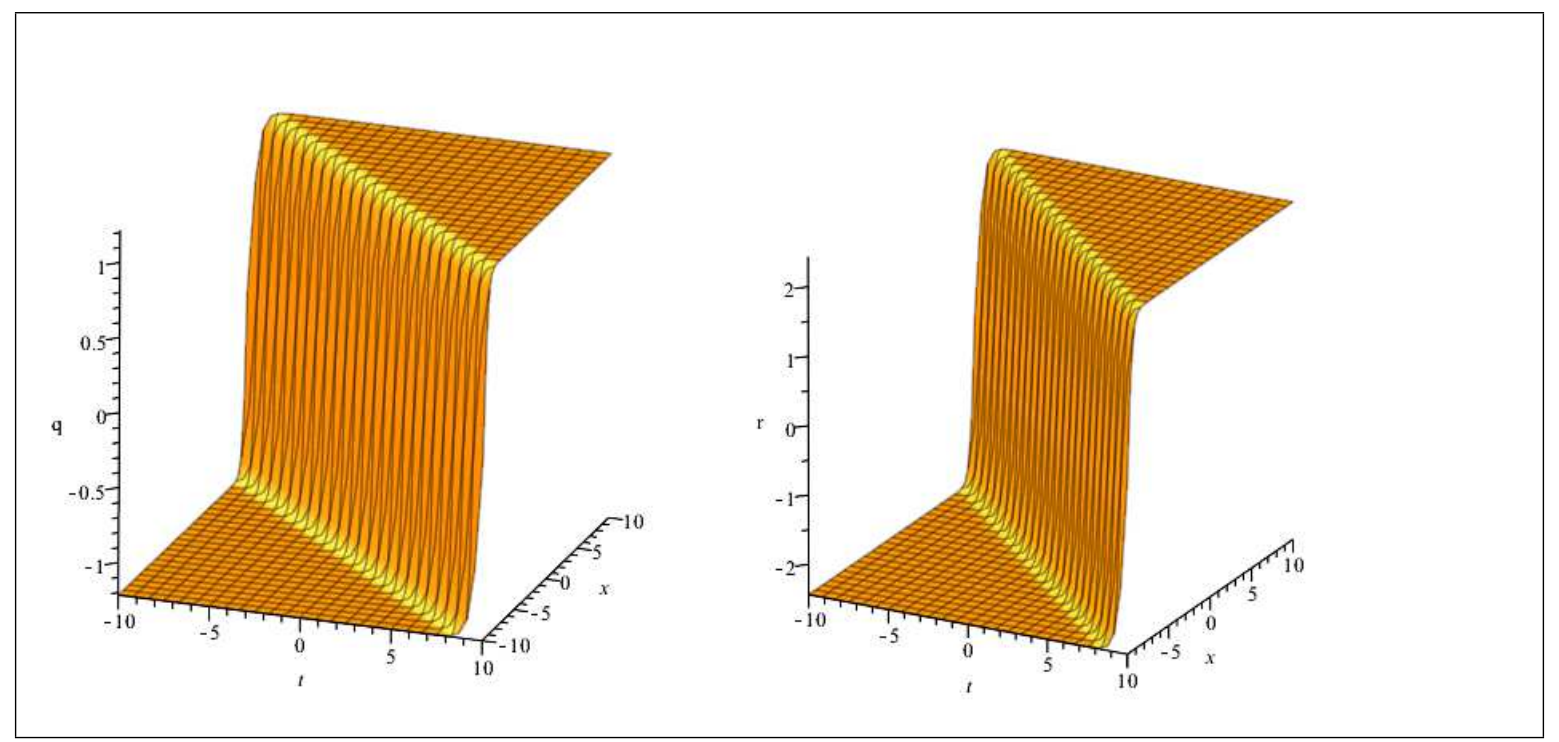

Figure-1

Figure-1. Plot of the dark soliton solutions (42) and (43) with $a_{1}=0.4, b_{1}=0.1, \kappa=1, \chi=2$, $\xi_{1}=0.2, \eta_{1}=0.3, \zeta_{1}=0.22, v=-1$ and $-10 \leq x, t \leq 10$.

Family-2. If $C_{0}=\frac{C_{4}^{3}}{32 C_{6}^{2}\left(1-m^{2}\right)}, C_{2}=\frac{C_{4}^{2}\left(4 m^{2}-5\right)}{16 C_{6}\left(m^{2}-1\right)}, C_{6}>0$, then

$$
u(x, t)=\left(\frac{6 L_{1}^{2}\left(m^{2}-1\right)^{2}}{L_{5}\left(2 m^{2}-1\right)^{2}}\right)^{\frac{1}{4}} \mathrm{nc}\left(\sqrt{\frac{L_{1}}{1-2 m^{2}}} \xi, m\right) \mathrm{e}^{i\left(-\kappa x+\omega t+\theta_{0}\right)},
$$

and

$$
v(x, t)=\chi\left(\frac{6 L_{1}^{2}\left(m^{2}-1\right)^{2}}{L_{5}\left(2 m^{2}-1\right)^{2}}\right)^{\frac{1}{4}} \mathrm{nc}\left(\sqrt{\frac{L_{1}}{1-2 m^{2}}} \xi, m\right) \mathrm{e}^{i\left(-\kappa x+\omega t+\theta_{0}\right)}
$$

or

$$
u(x, t)=\left(\frac{6 L_{1}^{2}}{L_{5}\left(2 m^{2}-1\right)^{2}}\right)^{\frac{1}{4}} \mathrm{ds}\left(\sqrt{\frac{L_{1}}{1-2 m^{2}}} \xi, m\right) \mathrm{e}^{i\left(-\kappa x+\omega t+\theta_{0}\right)},
$$

and

$$
v(x, t)=\chi\left(\frac{6 L_{1}^{2}}{L_{5}\left(2 m^{2}-1\right)^{2}}\right)^{\frac{1}{4}} \mathrm{ds}\left(\sqrt{\frac{L_{1}}{1-2 m^{2}}} \xi, m\right) \mathrm{e}^{i\left(-\kappa x+\omega t+\theta_{0}\right)},
$$

where $0<\left(1-2 m^{2}\right)<1$ and $L_{5}>0$. In particular, if $m \rightarrow 0^{+}$, in (48) and (49), then we have the periodic solutions:

$$
u(x, t)=\left(\frac{6 L_{1}^{2}}{L_{5}}\right)^{\frac{1}{4}} \sec \left(\sqrt{L_{1}} \xi\right) \mathrm{e}^{i\left(-\kappa x+\omega t+\theta_{0}\right)},
$$


and

$$
v(x, t)=\chi\left(\frac{6 L_{1}^{2}}{L_{5}}\right)^{\frac{1}{4}} \sec \left(\sqrt{L_{1}} \xi\right) \mathrm{e}^{i\left(-\kappa x+\omega t+\theta_{0}\right)},
$$

while if $m \rightarrow 0^{+}$, in (50) and (51), then we have the same periodic solutions (46) and (47).

\section{The addendum to Kudryashov's method}

According to this method $[2,19-21]$, we balance $P_{1}^{(4)}$ with $P_{1}^{5}$ in Eq. (31), we get

$$
M+4 p=5 M \Longrightarrow M=p .
$$

Now, the following cases can be considered.

Case-1. Choose $p=1$, then $M=1$. Now, we have the formal solution

$$
P_{1}(\xi)=B_{0}+B_{1} R(\xi)
$$

where $B_{0}$ and $B_{1}$ are parameters, provided $B_{1} \neq 0$. Here $R(\xi)$ satisfies the differential equation:

$$
{R^{\prime 2}}^{2}(\xi)=R^{2}(\xi)\left[1-\lambda R^{2}(\xi)\right] \ln ^{2} K, \quad 0<K \neq 1,
$$

where $\lambda$ is a constant. Substituting (55), (56) in (31) and setting all the coefficients of $[R(\xi)]^{u_{1}}$ $\left[R^{\prime}(\xi)\right]^{u_{2}},\left(u_{1}=0, \ldots, 5, u_{2}=0,1\right)$ to zero, we have the results

$$
B_{0}=0, B_{1}=\sqrt{\frac{2 \lambda\left(L_{1}+10 \ln ^{2} K\right) \ln ^{2} K}{\left(L_{4}+2 L_{2} \ln ^{2} K\right)}},
$$

and

$$
L_{3}=-\left(L_{1}+\ln ^{2} K\right) \ln ^{2} K, L_{5}=\frac{3\left(L_{1} L_{2}+2 L_{2} \ln ^{2} K-4 L_{4}\right)\left(L_{4}+2 L_{2} \ln ^{2} K\right)}{2\left(L_{1}+10 \ln ^{2} K\right)^{2}},
$$

provided $\lambda\left(L_{4}+2 L_{2} \ln ^{2} K\right)>0$. Substituting (57) along with the well-known solution of Eq. (56) obtained in $[2,19-21]$ in $(55)$, we have the solutions:

$$
u(x, t)=\sqrt{\frac{2 \lambda\left(L_{1}+10 \ln ^{2} K\right) \ln ^{2} K}{\left(L_{4}+2 L_{2} \ln ^{2} K\right)}}\left(\frac{4 S}{4 S^{2} \mathrm{~K}^{\xi}+\lambda \mathrm{K}^{-\xi}}\right) \mathrm{e}^{i\left(-\kappa x+\omega t+\theta_{0}\right)},
$$

and

$$
v(x, t)=\chi \sqrt{\frac{2 \lambda\left(L_{1}+10 \ln ^{2} K\right) \ln ^{2} K}{\left(L_{4}+2 L_{2} \ln ^{2} K\right)}}\left(\frac{4 S}{4 S^{2} \mathrm{~K}^{\xi}+\lambda \mathrm{K}^{-\xi}}\right) \mathrm{e}^{i\left(-\kappa x+\omega t+\theta_{0}\right)} .
$$

The solutions (59), (60) can be rewritten in the form:

$$
u(x, t)=\sqrt{\frac{2 \lambda\left(L_{1}+10 \ln ^{2} K\right) \ln ^{2} K}{\left(L_{4}+2 L_{2} \ln ^{2} K\right)}}\left[\frac{4 S}{\left(4 S^{2}+\lambda\right) \cosh (\xi \ln K)+\left(4 S^{2}-\lambda\right) \sinh (\xi \ln K)}\right] \mathrm{e}^{i\left(-\kappa x+\omega t+\theta_{0}\right)},
$$


and

$$
v(x, t)=\chi \sqrt{\frac{2 \lambda\left(L_{1}+10 \ln ^{2} K\right) \ln ^{2} K}{\left(L_{4}+2 L_{2} \ln ^{2} K\right)}}\left[\frac{4 S}{\left(4 S^{2}+\lambda\right) \cosh (\xi \ln K)+\left(4 S^{2}-\lambda\right) \sinh (\xi \ln K)}\right] \mathrm{e}^{i\left(-\kappa x+\omega t+\theta_{0}\right)},
$$

which represent the combo bright-singular soliton solution. In particular, when $\lambda=4 S^{2}$, we have the bright soliton solution:

$$
u(x, t)=\sqrt{\frac{2\left(L_{1}+10 \ln ^{2} K\right) \ln ^{2} K}{\left(L_{4}+2 L_{2} \ln ^{2} K\right)}} \operatorname{sech}(\xi \ln K) \mathrm{e}^{i\left(-\kappa x+\omega t+\theta_{0}\right)},
$$

and

$$
v(x, t)=\chi \sqrt{\frac{2\left(L_{1}+10 \ln ^{2} K\right) \ln ^{2} K}{\left(L_{4}+2 L_{2} \ln ^{2} K\right)}} \operatorname{sech}(\xi \ln K) \mathrm{e}^{i\left(-\kappa x+\omega t+\theta_{0}\right)},
$$

provided $\left(L_{4}+2 L_{2} \ln ^{2} K\right)>0$, while if $\lambda=-4 S^{2}$, we have the singular soliton solutions:

$$
u(x, t)=\sqrt{-\frac{2\left(L_{1}+10 \ln ^{2} K\right) \ln ^{2} K}{\left(L_{4}+2 L_{2} \ln ^{2} K\right)}} \operatorname{csch}(\xi \ln K) \mathrm{e}^{i\left(-\kappa x+\omega t+\theta_{0}\right)},
$$

and

$$
v(x, t)=\chi \sqrt{-\frac{2\left(L_{1}+10 \ln ^{2} K\right) \ln ^{2} K}{\left(L_{4}+2 L_{2} \ln ^{2} K\right)}} \operatorname{csch}(\xi \ln K) \mathrm{e}^{i\left(-\kappa x+\omega t+\theta_{0}\right)},
$$

provided $\left(L_{4}+2 L_{2} \ln ^{2} K\right)<0$. The solutions (59)-(66) exist under the conditions (58).

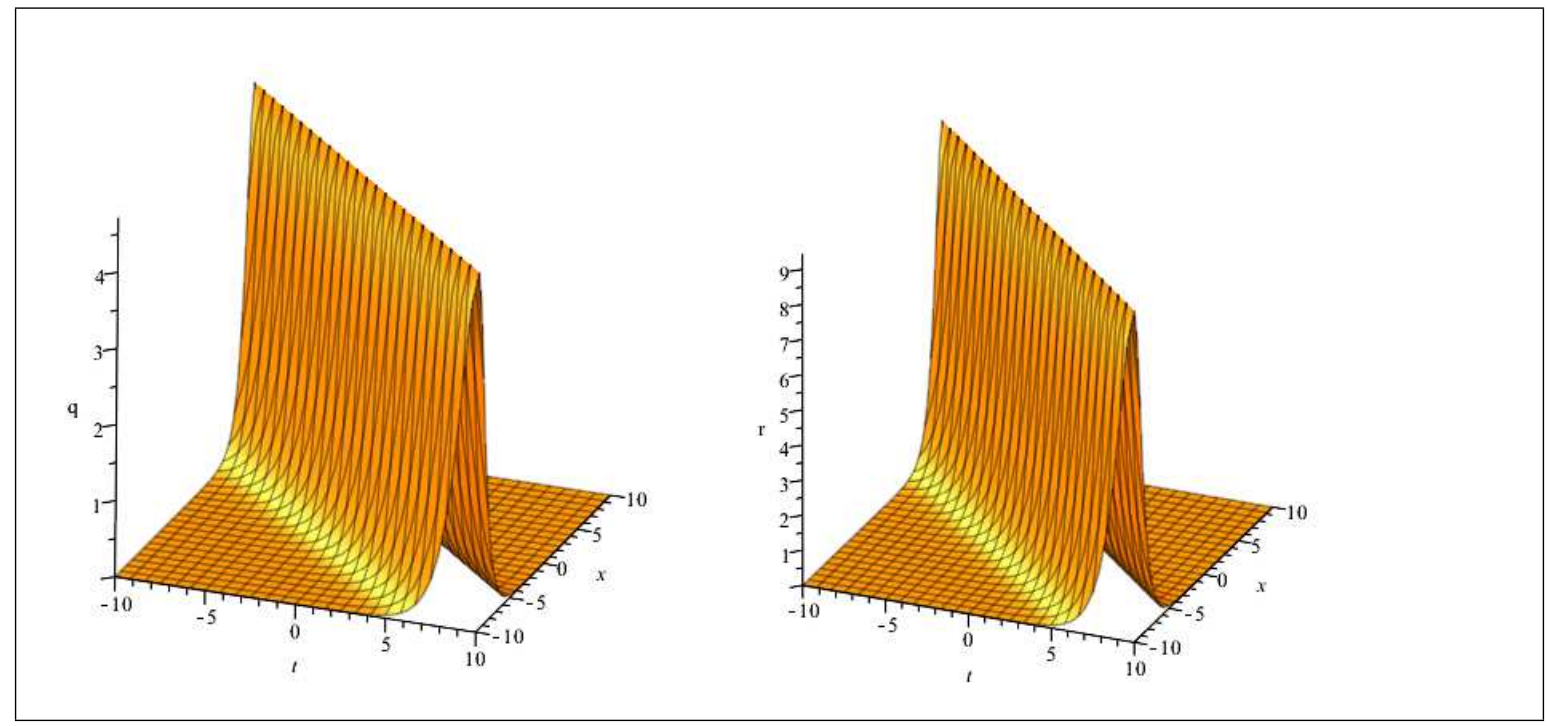

Figure-2

Figure-2. Plot of the bright soliton solutions (63) and (64) with $a_{1}=0.4, b_{1}=0.1, \kappa=1, \chi=2$, $\alpha_{1}=0.6, d_{1}=0.25, c_{1}=0.25, \gamma_{1}=0.2, \rho_{1}=-0.5, \sigma_{1}=0.33, v=-1, K=3, f_{1}=0.22, g_{1}=0.1$, 
$v=-1$ and $-10 \leq x, t \leq 10$.

Case-2. Choose $p=2$, then $M=2$. Now, we have the formal solution

$$
P_{1}(\xi)=B_{0}+B_{1} R(\xi)+B_{2} R^{2}(\xi)
$$

where $B_{0}, B_{1}$ and $B_{2}$ are parameters, provided $B_{2} \neq 0$. Here $R(\xi)$ satisfies the differential equation:

$$
{R^{\prime 2}}^{2}(\xi)=R^{2}(\xi)\left[1-\lambda R^{4}(\xi)\right] \ln ^{2} K, \quad 0<K \neq 1
$$

where $\lambda$ is a constant. Substituting (67) and (68) into (31) and setting all the coefficients of $[R(\xi)]^{u_{1}}\left[R^{\prime}(\xi)\right]^{u_{2}},\left(u_{1}=0, \ldots, 10, u_{2}=0,1\right)$ to zero, we have the results

$$
B_{0}=0, B_{1}=0, B_{2}=\sqrt{\frac{8 \lambda\left(L_{1}+40 \ln ^{2} K\right) \ln ^{2} K}{\left(L_{4}+8 L_{2} \ln ^{2} K\right)}},
$$

and

$$
L_{3}=-4 \ln ^{2} K\left(L_{1}+4 \ln ^{2} K\right), L_{5}=\frac{3\left(L_{1} L_{2}+8 L_{2} \ln ^{2} K-4 L_{4}\right)\left(L_{4}+8 L_{2} \ln ^{2} K\right)}{2\left(L_{1}+40 \ln ^{2} K\right)^{2}}
$$

provided $\lambda\left(L_{4}+8 L_{2} \ln ^{2} K\right)>0$. Now, we have the results:

$$
u(x, t)=\sqrt{\frac{8 \lambda\left(L_{1}+40 \ln ^{2} K\right) \ln ^{2} K}{\left(L_{4}+8 L_{2} \ln ^{2} K\right)}}\left(\frac{4 S}{4 S^{2} \mathrm{~K}^{2 \xi}+\lambda \mathrm{K}^{-2 \xi}}\right) \mathrm{e}^{i\left(-\kappa x+\omega t+\theta_{0}\right)},
$$

and

$$
v(x, t)=\chi \sqrt{\frac{8 \lambda\left(L_{1}+40 \ln ^{2} K\right) \ln ^{2} K}{\left(L_{4}+8 L_{2} \ln ^{2} K\right)}}\left(\frac{4 S}{4 S^{2} \mathrm{~K}^{2 \xi}+\lambda \mathrm{K}^{-2 \xi}}\right) \mathrm{e}^{i\left(-\kappa x+\omega t+\theta_{0}\right)} .
$$

The solutions (71), (72) can be rewritten in the form $u(x, t)=\sqrt{\frac{8 \lambda\left(L_{1}+40 \ln ^{2} K\right) \ln ^{2} K}{\left(L_{4}+8 L_{2} \ln ^{2} K\right)}}\left[\frac{4 S}{\left(4 S^{2}+\lambda\right) \cosh (2 \xi \ln K)+\left(4 S^{2}-\lambda\right) \sinh (2 \xi \ln K)}\right] \mathrm{e}^{i\left(-\kappa x+\omega t+\theta_{0}\right)}$,

and

$$
v(x, t)=\chi \sqrt{\frac{8 \lambda\left(L_{1}+40 \ln ^{2} K\right) \ln ^{2} K}{\left(L_{4}+8 L_{2} \ln ^{2} K\right)}}\left[\frac{4 S}{\left(4 S^{2}+\lambda\right) \cosh (2 \xi \ln K)+\left(4 S^{2}-\lambda\right) \sinh (2 \xi \ln K)}\right] \mathrm{e}^{i\left(-\kappa x+\omega t+\theta_{0}\right)},
$$

which represent the combo bright-singular soliton solution. In particular, when $\lambda=4 S^{2}$, we have the bright soliton solution:

$$
u(x, t)=\sqrt{\frac{8\left(L_{1}+40 \ln ^{2} K\right) \ln ^{2} K}{\left(L_{4}+8 L_{2} \ln ^{2} K\right)}} \operatorname{sech}(2 \xi \ln K) \mathrm{e}^{i\left(-\kappa x+\omega t+\theta_{0}\right)},
$$


and

$$
v(x, t)=\chi \sqrt{\frac{8\left(L_{1}+40 \ln ^{2} K\right) \ln ^{2} K}{\left(L_{4}+8 L_{2} \ln ^{2} K\right)}} \operatorname{sech}(2 \xi \ln K) \mathrm{e}^{i\left(-\kappa x+\omega t+\theta_{0}\right)},
$$

provided $\left(L_{4}+8 L_{2} \ln ^{2} K\right)>0$, while if $\lambda=-4 S^{2}$, we have the singular soliton solution:

$$
u(x, t)=\sqrt{-\frac{8\left(L_{1}+40 \ln ^{2} K\right) \ln ^{2} K}{\left(L_{4}+8 L_{2} \ln ^{2} K\right)}} \operatorname{csch}(2 \xi \ln K) \mathrm{e}^{i\left(-\kappa x+\omega t+\theta_{0}\right)},
$$

and

$$
v(x, t)=\chi \sqrt{-\frac{8\left(L_{1}+40 \ln ^{2} K\right) \ln ^{2} K}{\left(L_{4}+8 L_{2} \ln ^{2} K\right)}} \operatorname{csch}(2 \xi \ln K) \mathrm{e}^{i\left(-\kappa x+\omega t+\theta_{0}\right)},
$$

provided $\left(L_{4}+8 L_{2} \ln ^{2} K\right)<0$.The solutions (71)-(78) exist under the conditions (70).

Similarly, we can find many other solutions by choosing other values for $p$ and $M$.

\section{Conclusions}

Many exact solutions have been obtained for the coupled system of cubic-quartic nonlinear Schrödinger equation (CQ-NLSE) in fiber Bragg gratings with parabolic-nonlocal combo law of refractive index. The two integrated schemes, namely the extended auxiliary equation method and the addendum to Kudryashov's method are applied. The Jacobi elliptic function solutions, bright, dark and singular solitons from the governing model are obtained. These soliton solutions depend on certain constraint conditions and are given together with their existence criteria. Finally, our solutions have been checked using the Maple by putting them back into the original equations.

\section{References}

[1] N. A. Kudryashov. One method for finding exact solutions of nonlinear differential equations.Commun Nonlinear Sci Numer Simulat Volume 17, 2248-2253 (2012).

[2] N. A. Kudryashov. Method for finding highly dispersive optical solitons of nonlinear differential equations.Optik, Volume 206, 163550.(2020).

[3] N. A. Kudryashov. Highly dispersive optical solitons of equation with various polynomial nonlinearity law. Chaos, Solitons \& Fractals, Volume 140, 110202.(2020).

[4] N. A. Kudryashov. "Almost general solution of the reduced higher-order nonlinear Schr“odinger equation". Optik. Volume 230, 166347. (2021).

[5] N. A. Kudryashov. "Solitary waves of the non-local Schr" odinger equation with arbitrary refractive index". Optik. Volume 231, 166443. (2021). 
[6] N. A. Kudryashov. "Solitary waves of the generalized Sasa-Satsuma equation with arbitrary refractive index". Optik. Volume 232, 166540. (2021).

[7] A. Biswas, M. Ekici, A. Sonmezoglu \& M. R. Belic. Highly dispersive optical solitons with Kerr law nonlinearity by F-expansion. Optik. Volume 181, 1028-1038. (2019).

[8] A. Biswas, J. V. Guzman, M. F. Mahmood, S. Khan, M. Ekici, Q. Zhou, S. P. Moshokoa \& M. R.Belic. Highly dispersive optical solitons with undetermined coefficients. Optik. Volume 182, 890-896. (2019).

[9] A. Biswas, M. Ekici, A. Sonmezoglu \&M.R. Belic. Optical solitons in birefringent fibers with quadratic-cubic nonlinearity by extended $\left(G^{\prime} / G\right)$ - expansion scheme. Optik. Volume 178, 5965.(2019).

[10] A.Sonmezoglu, M. Ekici, M. Mirzazadeh, Q. Zhou, M. F. Mahmood, E. Zerrad, A. Biswas \& M. Belic. Optical Solitons in Cascaded System by Extended Trial Function Method.Comput\& Theoretical Nanoscience. Volume 13, 5394-5398. (2016).

[11] A. Bansal, A. Biswas, Q. Zhou, M.M. Babatin, Lie symmetry analysis for cubic-quartic nonlinear Schrödinger's equation, Optik, Volume169, 12-15. (2018).

[12] A. Biswas, H. Triki, Q. Zhou, S.P. Moshokoa, M.Z. Ullah, M. Belic, Cubic-quartic optical solitons in Kerr and power-law media, Optik, Volume144, 357-362. (2017).

[13] A. Biswas, A.H. Kara, M.Z. Ullah, Q. Zhou, H. Triki, M. Belic, Conservation laws for cubicquartic optical solitons in Kerr and power-law media, Optik, Volume145, 650-654.(2017).

[14] Y.Yıldırım, A.Biswas, A. J. M.Jawad, M. Ekici, Q.Zhou, S.Khan, A. K. Alzahrani, M. R.Belic, Cubic-quartic optical solitons in birefringent fibers with four forms of nonlinear refractive index by exp-function expansion. Results in Physics, Volume 16,102913.( 2020).

[15] G. Xu, Extended auxiliary equation method and its applications to three generalized NLS equations, Abst. Appl. Anal. 2014 Article ID 541370, pages 7. (2014).

[16] E.M.E. Zayed, R.M.A.Shohib, M. M. El-Horbaty, A. Biswas, M.Ekici, A. S. Alshomrani, F. B. Majid, Q. Zhou \& M. R. Belic. Optical solitons in birefringent fibers with LakshmananPorsezian-Daniel model by the aid of a few insightful algorithms. Optik. Volume 200, 163281. (2020).

[17] E.M.E. Zayed, M.A.Shohib, M. M. El-Horbaty, A. Biswas, M. Ekici , Q. Zhou, S. Khan , H. Triki, A. S. Alshomrani \& M. R. Belic. Dispersive optical dromions and domain walls with a few golden integration formulae. Optik. Volume 202, 163439. (2020).

[18] E. M.E. Zayed, M.E.M. Alngar, M. M. El-Horbaty, A. Biswas, M. Ekici, A. S. Alshomrani, S.Khan, Q. Zhou, M.R. Belic. Optical solitons in birefringent fibers having anti-cubic nonlinearity with a few prolific integration algorithms. Optik. Volume 200, 163229. (2020). 
[19] E. M. E. Zayed, T. A. Nofal, K. A. Gepreel, R. M. A. Shohib, M.E. M. Alngar, "Cubic-quartic optical soliton solutions in fiber Bragg gratings with Lakshmanan-Porsezian-Daniel model by two integration schemes", Optical and Quantum Electronics. Volume 53, 249 (2021).

[20] E. M.E. Zayed, K. A. Gepreel, M. E.M. Alngar, "Addendum to Kudryashov's method for finding solitons in magneto-optics waveguides to cubic-quartic NLSE with Kudryashov's sextic power law of refractive index", Optik. Volume 230, 166311 (2021).

[21] E. M.E. Zayed, R. M.A. Shohib, K. A. Gepreel, M. M. El-Horbaty, M. E.M. Alngar, "Cubicquartic optical soliton perturbation Biswas-Milovic equation with Kudryashov's law of refractive index using two integration methods" Optik. Volume 239, 166871 (2021).

[22] E. M.E. Zayed, R. M.A.Shohib, A. Biswas, M. Ekici, H.Triki, A. K. Alzahrani \& M. R.Belic. "Optical solitons with fiber Bragg gratings and dispersive reflectivity having parabolicnonlocal combo nonlinearity via three prolific integration architectures". Optik. Volume 208, 164065 (2020). 